\section{Resumen}

Individuo y sociedad son extremos de un continuo: hay procesos de "individuación" y de conformación de subjetividades sociales. Pero algunas explicaciones de la relación individuo-sociedad (individualismo metodológico, estructuralismo) han reducido el uno al otro. La idea de "masa" respondió a la posibilidad de transformaciones sociales en Europa; pero el análisis clasista de estos movimientos se mostró insuficiente. Ciertos autores "políticos", inquietos por el papel del sujeto en el cambio social, y ante la idea de una espontaneidad propia de la masa, se preguntaban: ¿Autodirección o partido? ¿Hay voluntad colectiva? ¿Es racional la masa? En esta línea, el oprimido debía ser transgresor (y, a más opresión, más reacción), y se tendió a subordinar el individuo a organizaciones burocratizadas. Los fracasos históricos llevaron incluso a ideas derrotistas del sujeto y del cambio. Pero otros autores (tanto "políticos" como "académicos"), al tener en cuenta las mediaciones (institucional, histórica, ideológica, valorativa, psicológica), pudieron entender la conformidad y la atomización del explotado, los efectos negativos de subordinar subjetividades individuales y de grupos primarios. Así, plantean la potencialidad del sujeto colectivo con distintas formas de expresión y de duración, en el marco de condicionamientos históricos específicos; y piensan, entonces, un sujeto en proceso, portador de conciencia, y agrupamientos no reducidos al determinismo económico.

\section{Palabras clave:}

Sociedad, sujeto, relación individuo-sociedad, cambio social, subordinación, subjetividad.

\section{Abstract}

Individual and society are extremes of a continuum: there are "individuation" processes and social subjectivity conformation processes. But some explanations of the individual-society relation (methodological individualism, structuralism) have reduced one to the other. The idea of "mass" responded to the possibility of social transformations in Europe; but the social class-based analysis of these movements proved to be insufficient. Some "politic" authors, concerned with the role of the subject in social change and face to the idea of the inherent spontaneity of the masses, questioned themselves: ¿Autodirection or party? $\dot{\text { Is }}$ there a collective purpose? $¿$ Is the mass rational? In this line of ideas, the oppressed one had to be the transgressor (and, the bigger the oppression, the bigger the reaction), and the individual was subordinated to bureaucratized organizations. The historic failures led to defeatist ideas of subject and change. But other authors (as much "politics" as "academics"), taking into account the mediations (institutional, historical, ideological, valuation, psychological), could understand the conformity and atomization of the exploited one, the negative effects of subordinating individual and primary groups subjectivities. Then, they state the potentiality of the collective subject with different forms of expression and duration, in a frame of specific historic conditions; and so they think of a subject in process, of a conscience carrier, and of groups unreduced to economic determinism.

Key words:

Society, subject, individual-society relation, social change, subordination, subjectivity. 


\title{
Pensar la sociedad y a los sujetos sociales*
}

\author{
Hugo Zemelman Merino'
}

\section{Un problema metodológico}

Uno de los problemas teóricos sustantivos de las ciencias sociales, a los cuales se ha conferido mayor importancia, es el de los sujetos sociales, independientemente de cuál sea su expresión histórica. Considerando los desafíos metodológicos del tema, no es necesario ahondar en detalles para señalar que hay dificultades metodológicas en el análisis de los actores, de los sujetos o de los movimientos sociales, y que éstas van más allá de su conceptualización. Dificultades que, en gran medida, se desprenden de dos desafíos que el tema contiene. El primero, el hecho de que tal problema es demasiado dinámico, y se agota sólo en la medida en que el investigador es capaz de estudiarlo en su propio movimiento; sin embargo, en la mayor parte de los estudios conocidos no siempre se ha logrado dicho propósito. El segundo, derivado del anterior, es la heterogeneidad de dinamismos que contiene la problemática del actor o del sujeto: se trata de una dinámica correspondiente no sólo a un plano de la realidad, sino a varios, incluso a planos de la realidad entrecruzados entre sí.

Para entrar en la cuestión con serenidad, convendría partir de una vieja problemática, ya registrada desde los clásicos, que no ha sido resuelta del todo, a pesar de los esfuerzos que ahora se emprenden para resolverla: me refiero a los últimos intentos de los llamados "individualismos metodológicos" que tie-

\footnotetext{
* Texto recibido en noviembre 15 de 2005 y arbitrado en junio 13 de 2006.

${ }_{1}^{1}$ Abogado y sociólogo. Actualmente se desempeña como presidente y director del Instituto Pensamiento y Cultura en América Latina, Ipecal. De 1974 a 2004 se desempeñó como profesor-investigador del Centro de Estudios Sociológicos de El Colegio de México. Hasta 1973 fue profesor del Departamento de Sociología de la Universidad de Chile, desempeñándose como director del mismo. Además de trabajar en El Colegio de México, se desempeñó como profesor en la División de Posgrado de la Facultad de Ciencias Políticas y Sociales de la Universidad Nacional Autónoma de México, Unam, así como en la Facultad Latinoamericana de Ciencias Sociales, de México. ipecal_mexico@yahoo.es
} 
nen su origen, fundamentalmente, en el mundo anglosajón, como respuesta a los enfoques llamados colectivistas o colectivo-estructurales. En el fondo, las perspectivas provenientes del individualismo metodológico son una respuesta a las dificultades enfrentadas por los análisis de clase según la convicción - como eje del debate- de que la perspectiva clasista ha resultado insuficiente para el estudio de los movimientos sociales. Por ello es indispensable saber hasta qué punto la categoría clase resulta insuficiente. Pero pienso que el fondo de tal cuestionamiento entraña una argumentación ideológica, sobre la cual quisiera abundar en el curso de esta exposición.

Durante el siglo XX observamos una diversidad de conceptos para dar cuenta de la problemática del sujeto. Originalmente, puede ubicarse una gran perspectiva teórica en el concepto de "masa" desarrollado en dos direcciones, casi en forma simultánea: como masa "espontánea" y como masa "unificada" en organizaciones. Éste fue un debate importante que se dio en los primeros quince años del siglo XX, y tuvo como telón de fondo los escenarios europeos, destacados por la expectativa de que en países muy desarrollados, como Alemania, se registraran revoluciones sociales. En ese lapso, tal debate representó un parámetro de la discusión por lo menos hasta 1920, en cuyo centro se planteaba la posibilidad de que ocurrieran grandes transformaciones en las sociedades europeas. El eje de la discusión giraba alrededor del concepto de masa: como masa espontánea, en la acepción de Rosa Luxemburgo, o como masa unificada en la organización, en el enfoque de Kautsky y Parvus, entre otros.

Avanzando en este panorama global, constatamos que el concepto de "masa" va tornándose más complejo, en función de situaciones históricas precisas. Esto produjo una serie de disquisiciones orientadas a rescatar la idea de sujeto, pero ya no sólo como el gran sujeto transformador, en la añeja visión del actor histórico que fue, por ejemplo, utilizada por Lenin y Rosa Luxemburgo (a pesar de sus distancias y sus diferencias, particularmente en lo relacionado con el concepto de masa). Lo nuevo consistió en que empezó a abordarse el problema del sujeto dentro de sus propias limitaciones estructurales, y surgió entonces una pléyade de conceptos a los cuales voy a referirme más adelante; entre otros, el concepto de "socialidad", propuesto recientemente por Maffesoli y que intentaría replantear el conjunto de la discusión contemporánea.

Esto nos induce a recorrer de nuevo estas divergencias conceptuales, tratando de seguir una línea de razonamiento en la búsqueda de una forma apropiada de estudiar al sujeto. Simultáneamente, se nos presenta otra vez un viejo desafío que tuvo siempre como trasfondo un cuestionamiento ya presente desde los clásicos: la relación entre individuo y sociedad. Sobre ese trasfondo se plantean los siguientes interrogantes: ¿En qué consiste la relación entre individuo y sociedad? ¿Es una dicotomía polar? ¿O habría que entenderla, más bien, como polos de un continuo? 
Si llevamos a cabo la relación entre individuo y sociedad en función de razonamientos dinámicos, evidentemente tenemos que tomar distancia de toda concepción dicotómica en el análisis de esa relación. Por tanto, se hace necesario incorporar la idea o, mejor dicho, el planteamiento metodológico que concibe la relación entre el individuo y la sociedad como dos polos extremos de un continuo. $\mathrm{Al}$ adoptar ese procedimiento, el primer paso consiste en preguntarnos respecto a la idea misma de continuo. Podemos encontrar distintas interpretaciones; no obstante, más allá de los matices, en conjunto, la pretensión central consiste (si se quiere resolver el problema y para no quedarse en la mera reducción de un polo en otro) en dar cuenta de la idea misma de continuo, negándose a optar por un discurso de reducción de la sociedad a lo individual (como es el caso de los individualismos metodológicos) o, inversamente, de reducción del individuo a la sociedad (tema recurrente en ciertos enfoques estructuralistas demasiado estáticos).

¿En qué consiste, pues, ese continuo? En la literatura se constata la existencia de dos conceptos (uno más elaborado que el otro) que, de alguna manera, pudieran estar dando cuenta de este proceso, de este continuo. Desde cierto punto de vista, la idea de continuo supone un razonamiento dinámico, apunta a un proceso complejo, constitutivo, diríamos nosotros; tal constitución se encamina en dos direcciones. Por una parte, hacia la dimensión del individuo, y puede llegar a expresarse en un concepto forjado por la psicología y retomado por ciertas corrientes antropológicas (Dumont). Me refiero al concepto de "individuación" y, en consecuencia, a su constitución; lo cual conlleva tanto un proceso constitutivo de la individuación como de lo colectivo, y se hace entonces más complejo el tratamiento del problema. Al respecto, conviene preguntarse: constitutivo, ¿de qué aspecto o ámbito social?, ¿de grupos, de clases, de etnias? Por otra parte, es precisamente aquí el lugar en el cual da inicio la discusión consistente en abrir el juego de las categorías, para dar cuenta de lo que estamos entendiendo por constitución de la dimensión social no reductible a lo individual. Desde ese marco, cabe recuperar la discusión forjada durante los últimos cien años - y aún no superada- en relación con las clases y las etnias; estas últimas recuperadas hoy, con gran fecundidad, en muchos análisis antropológicos, los cuales también han incorporado, en un plano metodológico, la categoría género.

En ese horizonte estamos quizás en presencia de un juego de categorías que nos habrá de conducir a dar respuesta a un problema relacionado con esa otra dimensión de lo constitutivo que es lo social y, por tanto, enfrentados a estos dos polos unidos por un continuo, como un proceso constitutivo que puede expresarse en individuación y en la conformación de ciertas subjetividades sociales. Es evidente que, al disponer del concepto de "subjetividad social", es necesario conducirse con cierta cautela, en tanto su acepción supone o eventualmente contiene una aporía. Sin embargo, esa es una parte de la discusión sobre la cual no es necesario detenerse en este momento. Lo que 
interesa dejar destacado es el juego de categorías que podría estar en marcha, e incluso disponible, en el recuento de esa segunda dimensión de lo constitutivo que es lo social.

Dicho en otras palabras: lo que se acaba de señalar es sólo un marco problemático en una primera aproximación. Para poder verlo con mayor serenidad y con mayor profundidad, sería necesario hacer un repaso muy detenido de la manera en que ha sido discutido el problema en los últimos cien años, algo que, obviamente, no puede hacerse en el marco del presente trabajo. Sin embargo, quisiera fijar algunas referencias o, mejor dicho, algunos hitos de referencia que son fundamentales (por lo menos desde 1900 hasta el presente), y para tal efecto elijo a cinco o seis autores que me han parecido relevantes; ello en un esfuerzo muy complejo y siempre inacabado de revisar esta reflexión que es tanto teórica como metodológica.

Voy a agrupar a estos autores en dos grandes categorías: un primer grupo constituido por que se caracterizan por llevar a efecto una reflexión de carácter político en los primeros treinta años del siglo XX (con excepción de algunos aportes de la psicología de masas), y un segundo grupo más cercano a los que organizaron los discursos académicos. Evidentemente, en ambas vertientes nos enfrentamos con discursos cuyas problemáticas, motivos y preocupaciones son de naturaleza diversa.

Los que aquí denominamos "autores académicos" trataron de encontrar una explicación a este continuo, mediante un recorte de observación, en un tiempo y en un momento histórico determinados. Algunos de ellos fueron más lejos: se plantearon la ambiciosa tarea de hacer recortes longitudinales, y llegaron con ello a establecer que la problemática de la constitución de lo social podría ser una clave para entender la evolución misma del ser humano, y se presenta como pensamientos a gran escala.

\section{Los autores políticos}

Inversamente, los autores que podrían ser caracterizados como políticos se propusieron resolver problemas no menos complejos, aunque en otra escala temporal. Éstos no buscaron hacer grandes interpretaciones acerca del papel, por ejemplo, de los sujetos en la historia o en la explicación de los procesos históricos; su pretensión fue, más bien, responder a la siguiente pregunta: ¿Qué hacer con los sujetos y qué pueden hacer los sujetos? Ésta es, sin duda, una discusión fundamental del siglo XX que, ideológicamente, se ha pretendido sepultar, calificándola de obsoleta. Sin embargo, la discusión se mantiene vigente hasta nuestros días, y quizá siga vigente durante el siglo XXI, toda vez que -y éste es un presupuesto- no declinemos de seguir pensando en el hecho de que el género humano continúe siendo responsable de sí mismo y de que en el futuro habrá de asumir, frente a sí mismo, su función de constructor. 
Por tanto, estas reflexiones respecto al siglo XX siguen siendo vigentes por varias razones. La primera de ellas (a pesar de todos los ajustes históricos que pueda tener la discusión) es que esos autores plantearon el problema del actor o del sujeto dentro de lo que sería una teoría del cambio social, no necesariamente regulado y marcado por leyes históricas, en la cual el acento se puso en la construcción de los propios actores. Ésta es una idea que estuvo muy presente en el marxismo clásico e incluso en el propio pensamiento bolchevique, aunque en este último con matices importantes. Para Bujarin, por ejemplo, el cambio del sistema económico y político no iba a ser el resultado fatal de las leyes internas del capitalismo, sino consecuencia de una acción colectiva consciente. Esa convicción, que estuvo en todo momento presente - en diversas formulaciones--, es lo que llevó a plantearse los grandes problemas teóricos con relación al sujeto.

\section{La "masa" según los autores políticos}

$\mathrm{Al}$ respecto, veamos algunos de estos planteamientos. El primero es el problema de la espontaneidad y, por tanto, el de la subjetividad como expresión de una espontaneidad colectiva o, para decirlo en términos de la época, el problema de la espontaneidad de la masa; una espontaneidad que se contenía a sí misma, esto es, que se autodirigía. El concepto de autodirección se refería a un tema que no hay que desechar, en tanto puede tener formulaciones actualizadas; su vigencia dependerá quizá de la significación conferida hoy al tiempo y al espacio social en el que se le ubique.

Así, por ejemplo, vale la pena diferenciar el pensar en masas espontáneas autodirigidas en la lógica de Rosa Luxemburgo (quien pretendía que esta masa fuera el gran actor de la transformación de la sociedad nacional y de la sociedad internacional) de lo que actualmente podríamos entender por espontaneidad autodirigida, cuya reflexión supone coordenadas de tiempo y de espacio mucho más restringidas, como se presentan ciertos tipos de movimientos locales en nuestra contemporaneidad. Aquí surge uno de los primeros problemas teóricos cuya duración nos alcanza: ¿Qué relación existe entre masa y organización? ¿Cómo puede resolverse dicha relación? Hasta el presente esta cuestión ha sido abordada a través de distintas formulaciones. Ejemplo de ello es el uso de una de las terminologías más usuales: la relación entre partido y movimiento, que ineludiblemente continúa siendo una discusión de actualidad (no obstante remontarse su origen a 1905 o quizás antes del siglo XX).

En el marco de dicha relación surge otro problema, relacionado con el continuo entre la individuación y los procesos de constitución de la subjetividad, y que se sintetiza en el concepto de "voluntad social". En apariencia, el concepto de voluntad tiene una génesis u origen psicológico, y puede estar vinculado más directamente al individuo; sin embargo, aquí no se trata de un planteamiento restringido al individuo, sino de una visión más vasta. Se trata de conferirle voluntad a un colectivo que 
puede surgir de una masa y que, al lado de esa voluntad atribuida, estaría presente un comportamiento subjetivo inherente a la masa misma. En otras palabras, se trata de identificar en la masa una concepción teleológica, una capacidad de plantearse finalidades y objetivos. Desde ese horizonte comienza a argumentarse que la masa espontánea es algo más que mera espontaneidad, y que es capaz de construir una organización de la voluntad con el fin de construir proyectos.

Ese es el centro de la discusión sostenida por Rosa Luxemburgo frente a Lenin y Kautsky, quienes, a pesar de sus diferencias, coincidieron en la defensa implacable de la voluntad organizadora de la masa; las diferencias apuntarían a lo que podríamos llamar - para usar tal vez un término más nuestro y actual- el ritmo de esa espontaneidad organizada. A pesar de que éstos son hechos generalmente conocidos y evidentes, hay en ellos un problema importante, que tiene que ver con ciertos colapsos que en nuestro tiempo hemos comenzado a vivir a partir de 1989, con el derrumbe del muro de Berlín.

Dos destacados autores en esta discusión, Pannekoek y Rosa Luxemburgo, eran intelectuales activos y no de escritorio, quienes, en su momento, establecieron un interesante vínculo teórico-político. Ellos conferían al concepto de masa una estructura racional y buscaban la función de esta estructura, pues en ella estaba contenido el espacio a través del cual habría de depositarse la esperanza del cambio. Esta idea puede trabajarse en muchas direcciones, aunque para nuestros fines podemos destacar dos: una en términos estrictamente ideológicos, que consistiría en apostar a la capacidad de esta voluntad social que se encuentra en la masa; y la otra, presente en la crítica de Karl Korch en 1922 (distinta de la orientación anterior), centrada en la idea de "potencia"; es decir, referida al problema de la esperanza en el cambio, que después sería objeto de distintos tipos de formulaciones, la última de las cuales estuvo presente durante la década de los años setenta del siglo XX en la versión desarrollada con una enorme fuerza por Ernst Bloch, mediante una invocación a la esperanza o, como se decía en esos años, por medio de una "convocación" a la misma.

Sin embargo, en los años veinte del siglo XX la idea de potencia no se trabajó como podría derivarse de la postura de Korch. Si rastreáramos un poco esta idea de potencia, es posible encontrarla en algunos de los pocos escritos que sobre el problema de la organización política redactó Georg Lukács cuando era dirigente político activo en esos años. Como quiera que fuere, lo que interesa destacar es la manera en que empieza a transformarse esa masa en una estructura que contiene la esperanza de cambio; es decir, el concepto de masa con esperanza.

De cualquier forma, hay que mantener con reserva esta idea de masa con esperanza. Al respecto, sería muy interesante confrontar este planteamiento que he sintetizado con el texto Masa y poder, de Elías Canetti. En la visión de Canetti aparece, de manera muy aguda y con muchas ejemplificaciones históricas, el concepto de masa; él aplica este concepto a determinados tipos de actores sociales 
o de movimientos sociales de inspiración escatológica o religiosa. Ello tiene que ver con uno de los grandes problemas que se han observado posteriormente: si esa masa ya no puede tener un comportamiento autodirigido, de acuerdo con la crítica de Kautsky dirigida a Rosa Luxemburgo (y que alcanza a casi todos los dirigentes posteriores, con excepción de los anarco-sindicalistas), entonces el problema de la autodirección se vincula con la siguiente cuestión que retoma después Jean-Paul Sartre, en el contexto europeo de los años cincuenta y sesenta del siglo XX: me refiero al hecho de que tal organización debilita la fuerza de la masa misma, con lo cual ésta deja de ser depositaria de toda esperanza de cambio.

La idea de Sartre tiene afinidad - aunque no en la misma línea de pensamiento- con los actuales planteamientos de Alberoni, con relación al problema existente entre masa, espontaneidad y organización; o, para decirlo en términos weberianos, entre lo que podríamos llamar una cierta fuerza carismática que podría expresar una esperanza de cambio, junto a la rutinización inevitable propia de la institucionalización, de la cual indudablemente forma parte el partido político.

\section{Efectos del reduccionismo}

Desde luego, esta línea de razonamiento no está agotada, ni mucho menos. Pero, por su misma complejidad, el problema tiende a resolverse en forma simplista e inmediatista. Ante situaciones en apariencia irresolubles, generalmente hubo una gran dificultad en el manejo de la relación entre todos los conceptos o de todos los problemas, con la tendencia siempre a resolver y a dar soluciones a los dilemas de dichas relaciones, durante todo el siglo XX (por lo menos hasta la gran producción académica a la cual apenas me he referido), desde el espacio del discurso político, incluido el discurso político de América Latina de los últimos veinte años.

En efecto, las soluciones han tendido a reducir, a subordinar el individuo al colectivo, como fue el caso del pensamiento político marxista o, mejor dicho, de una suerte de pragmática marxista en política. Al respecto, lo que importa no es discutir el carácter pragmático de las respuestas ofrecidas (y que son de todos conocidas), sino más bien encontrar y descifrar el razonamiento inherente a dichas respuestas.

Tal razonamiento que fundamenta la reducción del individuo al colectivo propició el surgimiento -en América Latina- de un fenómeno aún más grave: la subordinación de los movimientos sociales, mediante distintas formas de presión, a voluntades orgánicas que muchas veces se burocratizaban, casi sin excepción. Este era, desde luego, un razonamiento histórico; no obstante, se trata de saber en qué consistía tal razonamiento. Se llegó a considerar que el ser humano, en todo momento, inevitablemente - $\mathrm{y}$ sin mediar ningún espacio-, era un producto de las circunstancias sociales, antes que el resultado de un cierto mecanicismo de lo que los italianos mucho después, al estilo de Marramao, llamaron de una manera muy aguda el cartesianismo obrero. 
Lo dicho equivale a plantear que, por el hecho de estar sometidos a determinados condicionamientos económicos, a cada individuo puede atribuírsele una conciencia determinada. Esa era pues la premisa fundamental: dada determinada condición de explotación, de subalternidad o marginalidad, el efecto que de ahí se deriva consiste en el hecho consumado de que, quien es objeto de esa condición, tiene que ser por necesidad un crítico o transgresor de lo existente. Sin embargo, la historia muestra que las cosas no son así. Los factores económicos pueden ser una regularidad explicativa de muchos otros fenómenos, por ejemplo, de carácter cultural, político, ideológico o incluso psicológico, pero sólo en ciertos rangos de tiempo, en ciertas escalas de temporalidad; de modo que la premisa podría ser verdadera en la gran escala del tiempo y, probablemente, no serlo en escalas más cortas.

Razonar en las pequeñas escalas de tiempo, con ayuda de esta lógica tan determinista como la que estoy tratando de ejemplificar, condujo de modo inevitable a errores. Un error típico es el de pensar que cuanto peor sea nuestra condición, mejores perspectivas tendríamos ante nosotros. Para tal razonamiento, cuanto más deterioradas fueran las condiciones de vida de las masas, más tendrían éstas que reaccionar. Sin embargo, sabemos que en la realidad las cosas no son así ni siempre fueron así. Entre las condiciones económicas y el comportamiento humano existen mediaciones en extremo complejas de diversos tipos: institucionales, históricas, ideológicas, valorativas e incluso psicológicas; mediaciones que pueden alterar, por ejemplo, esa relación que se plantea como cartesianismo obrero, y que ha sido objeto de crítica por parte de autores como Marramao, entre otros.

Lo que tenemos no es una relación simple de causa-efecto. Antes bien, hay en esa relación un fenómeno complejo que contiene una gran variedad de mediaciones; y precisamente, en virtud de su presencia, sobre todo en el surco entre el factor determinista de carácter estructural y el comportamiento individual o grupal, es desafortunado que dichas mediaciones no estuvieran presentes en el análisis de clase. Desde luego, esto no significa que la categoría clase no tenga peso ni relevancia; inversamente, lo que no es importante y relevante es el razonamiento mecánico formulado desde la clase que deja en un universo de sombras la cuestión de las mediaciones.

Así, por ejemplo, es más fácil razonar la clase en términos reduccionistas que complicarse el problema preguntando: ¿Qué hay entre la clase y el individuo? ¿Qué se deja ver entre la clase y el grupo primario? ¿Qué es posible observar entre la clase y los grupos secundario o terciario? Esta forma de plantearse los problemas es un proceder más complejo que cierra el paso a un dispositivo por reducción a lo simple, y permite con ello conferir un sentido riguroso a la categoría clase que permitiría responder con mayor precisión al problema que nos hemos planteado

Esta es una vieja discusión en la cual, por el momento, no quisiera adentrarme, pues hay otra de gran relevancia que es crucial en el presente y que, sin duda, 
merece nuestra atención: se trata de la reducción de lo colectivo al individuo, y en cuyas expresiones metodológicas actuales (en el ámbito de las ciencias sociales) se han realizado trabajos importantes, por lo menos en ciertas vertientes de la sociología de los movimientos sociales, sintetizadas en los llamados individualismos metodológicos. Quienes han sostenido tal planteamiento, a veces se dan cuenta de que están cometiendo algunos desaciertos, y buscan de inmediato inculpar de ello a Max Weber. Ello resulta complicado, si consideramos la impostura de fijar un planteamiento no del todo correcto, y que no es asumido en forma clara de modo suficiente. Así, al constatar que el problema no termina de resolverse, en tanto no se considere la problemática del continuo entre estos dos polos (la dinámica entre individuo y sociedad), se torna evidente la necesidad de retrotraernos a las preguntas inicialmente planteadas: ¿Qué une a esos dos polos en términos dinámicos? ¿Por qué no hay ahí ningún cordón umbilical? Sin duda, estamos ante un proceso cuya complejidad supone un gran esfuerzo de desciframiento. A tal proceso hemos estado dándole un nombre sin precipitar, desde luego, un juicio teórico al respecto: nos referimos a los procesos constitutivos.

En efecto, la idea de que ese continuo entre individuo y sociedad -para decirlo en términos temáticos- es un proceso constitutivo, supone precedentes históricos que deberían ser suficientemente recuperados. En relación con ello, hay varias ideas: por ejemplo, el concepto de "lucha" (Lagarde o Preobrazhensky), que, en forma evidente, no se agota en la pura reivindicación gremial, económica, política o cultural; antes bien, el concepto de lucha alude a una dinámica mucho más profunda, que podría entenderse como una dinámica constitutiva, y que hizo necesario plantear la idea de sujeto como proceso.

Estamos hablando de planteamientos que tienen algo más de cuarenta años, recuperando de ellos líneas que han quedado sueltas y perdidas en el tiempo, pero que indiscutiblemente forman parte de la acumulación de problemas consustanciales al debate durante el siglo XX. Una de esas líneas es el concepto de "sujeto como proceso", del cual se ocuparon diversos autores -según distintas perspectivas-, por ejemplo, Wilhelm Reich. La idea de sujeto como proceso está emparentada con grandes problemas del presente vinculados a la llamada crisis de las utopías, y que supone un desafio que ya había sido desarrollado desde entonces. En el fondo de tal desafio hoy está la recurrente creación de nuevos sujetos sociales, lo cual torna necesario no limitarse a una mera descripción de tales emergencias -en el sentido luhmanniano del término- que ha aprisionado al pensamiento en lo dado, y que lo inhabilita para reflexionar más allá del limitado horizonte de la descripción.

Contrariamente, en el presente se trataba de ver (como lo sugirió después de la gran tradición Fernando Claudin para el contexto español) la problemática de los sujetos limitados, sacrificados, incluso derrotados. Ese fue un diagnóstico histórico elemental que requería plantear el problema de los sujetos en función de "nuevos" sujetos sociales, confiriéndoles el carácter de fuerzas morales del futuro. 
Este problema surgió en la década de los años veinte del siglo XX y fue percibido de manera clara e intangible como una gran expectativa, y fue motivo de reflexión para el pensamiento crítico desde fines del siglo XIX, con la influencia muy directa de Marx y de los anarquistas de la época como Blanqui.

También es una referencia importante el gran pensamiento teórico socialdemócrata europeo durante los primeros quince años del siglo XX, para el cual era problemática la universalidad de la revolución, principalmente a partir de 1918 y 1919, con las derrotas de las llamadas comunas de Viena y sobre todo de Berlín. Algo muy parecido ocurre luego en la Italia teorizada por Gramsci, y en la cual también se experimentó, en los años veinte, una gran derrota; ahí el paradigma se derrumbó en forma inevitable al no surgir los grandes cambios en los países desarrollados, anticipados por el paradigma gramsciano. La reacción de esas generaciones fue iniciar el planteamiento del problema de lo que, textualmente, llamaron las fuerzas morales del futuro; es decir, de los actores y sujetos, que no se habían aún hecho presentes, por lo cual era necesario y urgente contribuir a su potenciación y su construcción.

Tal idea es congruente con un planteamiento después olvidado y sepultado por razones muy claras; Bujarin, por ejemplo, sostenía que el capitalismo no se autoderrotaba (idea que Lenin llegó a compartir), que no se derrumbaría sólo como resultado de sus contradicciones internas; según esa óptica, lo que se quería refutar era la idea de una supuesta ley inexorable del derrumbe del capitalismo. Quizá por entonces podría haber ocurrido algo muy parecido a aquel supuesto que ocasionalmente aún tiene resonancia en el presente: creer que los cambios advienen por sí mismos, sin que en ellos medien voluntades sociales organizadas; de ahí la importancia -llamémosle "ontológica" - que tuvo el partido político, así como la idea de proyecto, como presupuesto para la construcción de la crisis y, por tanto, para la construcción de alternativas.

Ahora bien, es necesario no olvidar que lo ocurrido en Alemania, con la frustración de las grandes experiencias revolucionarias y con el advenimiento del fascismo, vino a complejizar todavía más el problema del sujeto. En el marco de ese contexto surgieron diversas teorizaciones, entre ellas la de Reich, que comenzaron a fijar su atención en otros problemas, que ya no sólo tenían que ver con ese mecanicismo inherente al determinismo económico por entonces dominante. Comienza entonces a trabajarse con mayor atención un problema que, en esos años, es visto con mayor claridad y que, de manera simultánea, era resultado de esas coyunturas históricas. Me refiero a la incorporación de la siguiente idea: la economía no lo explica todo; es decir, lo económico no necesariamente construye un edificio congruente con sus propias exigencias. Esa construcción edificada por la economía entraña problemas internos: fue hecha por varios arquitectos que, desde el principio, estaban enfrentados. Mientras el piso de la economía tendría una dimensión, al piso de la ideología se le atribuirían otras coordenadas. Al res- 
pecto, no parece que estemos ante un edificio muy armonioso; lo que más bien observamos es una suerte de anticipo de la arquitectura de Gaudí.

A partir de esa premisa sencilla, se empezó a tomar conciencia de la necesidad del análisis de esas divergencias existentes entre los distintos pisos de la construcción o, por decirlo de manera simple, entre economía e ideología. Sobre la base de esa toma de conciencia, la discusión se desplazó hacia lo que, en sentido teórico, se ha dado en llamar, desde hace cincuenta años, la aconflictividad interna de un actor, y que equivale a analizar, de una manera no cartesiana -para decirlo en términos de Marramao-, la relación entre situación social y actitudes de cambio. En este asunto surge la siguiente cuestión, obvia en apariencia, pero que de pronto cobró relevancia en las discusiones políticas en América Latina: dados ciertos condicionamientos, por ejemplo, de carácter ocupacional, o dada la presencia de ciertas pautas ideológicas que fueron socializadas, o para decirlo en términos mertonianos, dados ciertos grupos de referencia, ¿puede atribuirse, a un individuo o a un grupo primario, una disposición (que no necesariamente va a imponerse) hacia el cambio?, ¿es acaso pertinente tal atribución, cuando es adverso el contexto (llamado en su momento "atmósfera social") en el que están inmersos ese individuo o grupo?

En efecto, a la idea de atmósfera social podrían incorporarse problemas contextuales. Un obrero, un campesino o un integrante de las capas medias, es posible que esté en desacuerdo con su situación; no obstante, un razonamiento no cartesiano obliga a considerar el contexto o atmósfera social en el que vive dicho individuo. Ejemplo de ello es la gran fuerza que hoy ostenta el discurso neoliberal y que directamente apunta a esa atmósfera, y se traduce en actitudes y posturas muy concretas, como serían: el desinterés del individuo por la política y la creencia - que se piensa es positiva-de que otros practiquen la política en su lugar; la convicción de que no es posible cambiar nada, en la medida en que todo permanece inamovible.

Esos son, precisamente, síntomas de cierta presencia contextual sobre una subjetividad que puede ser incongruente consigo misma, que, sin embargo, termina por doblegarse. Se puede también mencionar, a manera de ejemplo, una situación producida en la Europa de los años veinte del siglo XX, en ocasión de la frustración de los proyectos revolucionarios en varios países: movimientos encabezados por huelgas generales impactantes, como las que se dieron en Inglaterra y Bélgica, o como las insurrecciones obreras en Viena y en Berlín. La razón del advenimiento de esos fracasos tiene que ver con una serie de mecanismos inherentes a la compleja relación de la subjetividad individual, o de la subjetividad que puede surgir en un grupo primario, respecto a la subjetividad colectiva que termina por imponerse sobre aquella.

A partir de este contexto histórico europeo, tiempo después -siguiendo la línea del pensamiento de Gramsci- dan comienzo algunas teorizaciones de gran 
interés sobre el problema del sujeto. ¿A qué obedece el que Gramsci incorporara la cultura de manera sistemática? ¿Por qué a Gramsci le preocupó la cultura de Italia, y en qué sentido teorizó, por vez primera, las formaciones económicas y sociales, y complejizó la vieja fórmula de Marx con el problema de la división cultural? La razón fundamental está en que Gramsci percibió y quiso reaccionar frente al fenómeno del dominio impuesto sobre los sectores subalternos, en tanto dicho dominio iba más allá de sus condiciones de trabajo y de su explotación, a través de patrones ideológicos y culturales, lo cual Gramsci designó con el término de "subalternidad". La subalternidad representa la gran conquista de todo régimen: mediante ella se logra que los marginados y los explotados estén conformes de serlo. La subalternidad plantea un problema que no es, en sentido estricto, propio del ámbito de la tecnología o de la economía; tiene más bien que ver con un ámbito de mayor alcance, como es el de la cultura.

\section{Potencialidad}

Desde entonces han surgido teorizaciones al estilo de Rossana Rosanda, de Lucio Magri y muchos otros, quienes comenzaron a poner mayor atención en lo que había sido -en ciernes- un concepto no incorporado suficientemente en el análisis del sujeto y de las subjetividades sociales: me refiero al concepto de potencialidad. No en un sentido abstracto, sino como potencialidad recuperada; es decir, como la potencialidad de una subjetividad colectiva con distintas formas de expresión organizativas, con diversas maneras de expresión en sus prácticas sociales, con diferentes duraciones temporales (y éstas pueden ser coyunturales o de mayor alcance).

Esa es, pues, la potencialidad en el interior de los condicionamientos históricos; es el análisis de la potencialidad (para que se comprenda mejor) en el encuadre político partidista y no partidista, y abierto, por tanto, a otros encuadres posibles, como podrían serlo otras tantas formas de expresión de los actores, vale decir, de los movimientos sociales. Aquí surge una gran advertencia ya contenida en la crítica gramsciana a los bolcheviques: no es posible restringir la fuerza de esa masa organizada o espontánea (para retomar el viejo concepto luxemburguista) al encuadre estricto de una forma particular de sus expresiones, como pudiera ser, por ejemplo, el partido político.

Lo que en ese debate se discutía tiene hoy una gran presencia, y nos conduce, sin duda, a plantear un problema que en este momento es menester recuperar, pues viene arrastrándose a partir de esta genealogía. Me refiero a algo que ya habíamos anticipado: la relación conflictiva entre dinamismos sociales y estructuras institucionales. Fue Sartre quien percibió este problema al formular una pregunta a la cual intentó responder ulteriormente Alberoni (aún sin ser sartreano) en su texto sobre Movimientos e instituciones. Esa pregunta -en cierta medida también de inspiración weberiana- se formula así: ¿Cómo lograr la persistencia de la energía 
o de la fuerza de la masa sin que se desgaste en las instituciones? La cuestión apunta, en términos weberianos, a la relación entre carisma y rutinización burocrática. Para decirlo en forma un tanto exacerbada, diríamos que el problema de dicha relación surge, precisamente, a partir del rescate de una dimensión cualitativa en el análisis de los sujetos, teniendo, como experiencia central, las derrotas obreras experimentadas en Europa y, como antecedentes principales, el fracaso del movimiento comunista italiano frente al fascismo, así como el hundimiento del Partido Comunista y del Partido Socialdemócrata alemanes frente al nacional-socialismo. Las reacciones ante esas experiencias fatales emergieron en la segunda posguerra por una razón simple: durante el conflicto bélico no había tiempo para la reflexión de esos sucesos históricos; no obstante, en esos años se registran algunas aportaciones interesantes que habría que revisar; me refiero, por ejemplo, a los textos de políticos pragmáticos como Togliati que después fueron acusados de estalinistas..., pero ello merece una reflexión que rebasa un texto como éste.

A lo anterior puede agregarse otro problema que es interesante plantear y que, por su relevancia enorme, comienza a transformarse en el nuevo eje articulador de la problemática sobre sujetos sociales. El problema es el siguiente: desplazar el eje de la discusión del ámbito definido por las condiciones estructurales (entendiendo por ello los condicionamientos económicos o institucionales) hacia otro que, en lo sucesivo, jugará un papel diferente. Se trata del eje de la conciencia y su lugar frente a esas condiciones estructurales. Esta es un cuestión que está presente hasta nuestros días, por ejemplo, en la acepción de Lucio Magri, quien formuló una idea tan simple que, quizá por ello, no la terminamos por asimilar: plantear el hecho de que la crisis del capitalismo no es fundamentalmente una crisis económica, sino una crisis social e ideológica, y el partido adviene tanto en un factor de poder como en una expresión ideológica.

Tras estas frases no se apunta de manera exclusiva a una experiencia anterior, que es la que estamos tratando de resumir y recuperar, sino también a una nueva manera de entender el problema del sujeto, mediante el cual el partido deviene - a la luz de este marco de discusión- no sólo en mero instrumento de las acciones de un sujeto histórico preexistente. Esta acepción de "preexistente", en gran medida predominante hasta después de la segunda mitad del siglo XX, es fundamental en tanto que detrás del rescate del partido, el sujeto preconstituido estuvo presente durante un largo período en las formas hegemónicas que revistió el análisis de los sujetos. Esta idea se había hecho presente en la construcción del partido, atribuyendo a éste un carácter cuasiontológico. También está presente otra idea que es curiosamente contradictoria: me refiero a la construcción del derrumbe (para decirlo según Colletti), cuyo fin era no esperar el derrumbe, en los términos de una teoría según la cual estarían contenidos antes, en sí misma, los mecanismos del derrumbe; ahí la perspectiva del sujeto preexistente en la construcción del derrumbe, suponía un cambio en las formas de su acción, que 
inducía a transformar aquello que necesariamente debería ser derrumbado.

En América Latina, durante la década de los años sesenta y setenta del siglo XX, este personaje colectivo (el sujeto del partido) era también una entidad preexistente; el discurso político le atribuía características y fines definidos previamente. El sujeto no era susceptible de experimentar la influencia de ningún tipo de mediación. Después, la idea que comienza a retomarse (de hecho ya se había introducido tiempo atrás) es la de un sujeto entendido como proceso, acepción cuya procedencia se remonta a ciertos analistas italianos y a la cual habría de reincorporarse de manera creativa, en los estudios latinoamericanos, la problemática de la conciencia.

Se empezó pues a analizar al sujeto partiendo de dos parámetros, en forma similar al análisis dominante de un discurso cuya procedencia se remonta al marxismo clásico de comienzos del siglo XX. El sujeto es concebido como un proceso y, al mismo tiempo, aparece como portador de conciencia. Por tanto, el partido es una forma privilegiada de expresión ideológica para el sujeto; pero si ese proceso no se conduce de manera congruente, se corre el riesgo de caer, de nueva cuenta, en la dogmatización del sujeto. Estas dos acepciones no fueron respetadas en forma consecuente. Quizá la excepción a la regla estuvo representada en la experiencia del Partido Comunista Italiano. Esas dos lecciones no fueron respetadas esencialmente en el plano del discurso político, a pesar de la presencia del sujeto como proceso y del rescate de la problemática de la conciencia (después reforzada con la crítica del socialismo real). El partido continuó siendo reducido a un mecanismo operativo del poder, al frente de un sujeto preexistente, inmutable e inmodificable. Éstas son, en síntesis, las incongruencias del mundo político; ulteriormente mostraremos la necesidad y la pertinencia de observar las del mundo académico.

La idea del sujeto-proceso y la cuestión de la conciencia, se vincula con un problema que es importante aclarar: al pensar el sujeto y sus formas de expresión en términos dinámicos, quizá sea posible rescatar las potencialidades del mismo, en particular su subjetividad en el interior de sus condicionamientos históricos. De hecho, esta tarea no se ha emprendido; su dilucidación depende de una dialéctica de los condicionamientos históricos y, por tanto, de las limitaciones históricas de naturaleza múltiple que se presentan ante un actor social constituido que, al disponer de ciertas formas de organización, se plantea proyectos ante sí.

Este tipo de dificultades no siempre se tienen presentes, en tanto no se considere, en el análisis del sujeto, el problema de su correlato; es decir, el de las dificultades externas al sujeto y, simultáneamente, el de sus propias dificultades internas. Ese es el punto fundamental en el cual surgen también consideraciones de carácter ideológico, a partir de las cuales es indispensable reconocer los obstáculos que enfrenta el sujeto; en otras palabras, el momento en que tiene que considerarse, en su dimensión real, la idea del sujeto como proceso que, desde el punto de vista de la construcción social, supone la constitución del sujeto mismo. 
Esta problemática está contenida en el interior de las limitaciones del sujeto, y su comprensión es más compleja en tanto más grandes son las barreras impuestas a un sujeto. El problema podría ejemplificarse con mayor claridad en el ámbito de los sistemas represivos; por ello, no es extraño que ese tema surgiera por ejemplo, en España, a partir del análisis del franquismo por parte de un autor como Claudin, y, en Alemania, desde la crítica al socialismo real emprendida por Rudolf Bahro. Ambos autores, con signos distintos, asumieron el desafío de la construcción del sujeto ante sus propias dificultades. En su momento, Bahro planteó el problema de enfrentar como un gran obstáculo (que se vincula, desde luego, con el papel de la conciencia), en el plano de la subjetividad de los actores sociales, lo que él llamó la subalternidad derivada de la división del trabajo: el fraccionamiento y la pasividad provenientes de una suerte de atomización del actor, como un producto de la división del trabajo. Bahro teoriza sobre la necesidad de rescatar la idea de sujeto como proceso y en ella la función conferida al tema de la conciencia, cuyo papel es dar cumplimiento a la integración del sujeto y crear las condiciones para su participación. Así, formuló una serie de consideraciones respecto a dos tipos de conciencia, con relación a la subjetividad del sujeto: la conciencia absorbida y la conciencia excedente. La conciencia absorbida opera tanto en la dimensión del individuo como en el plano de las diferentes formas de expresión de lo colectivo. Ese tipo de conciencia supone un gasto de energía o fuerza (que pudiera ser psicosocial) y responde, por decir lo menos, a una política de cortoplacismos: disputas de puestos y cargos, respeto de las jerarquías, etcétera. Desde luego, éste es un tema extremadamente interesante que se expresa, para decirlo de manera weberiana, en las actividades de la vida diaria y, por tanto, en la reproducción rutinaria que va conformando la masa subalterna, teniendo -según Bahro-, como trasfondo de esa conciencia absorbida, la división del trabajo. En cambio, lo que importa a la conciencia excedente que presupone toda esa fuerza y esa energía, es que no está sometida -y éste es un punto fundamental- a ninguna de las limitaciones ni las deformaciones de las divisiones o de los tipos de trabajo.

En estas consideraciones sobre la cuestión de la subjetividad hemos centrado nuestra reflexión en el discurso político. Ahora es conveniente preguntarse cómo se ha abordado la problemática de la subjetividad en el discurso académico.

\section{Los autores académicos}

El discurso académico es igualmente amplio y tiene una historia tan larga como la del discurso político. Tiene autores muy relevantes que, desde diferentes perspectivas, apuntan al proceso que hemos intentado analizar en el discurso político; incluso me atrevería a afirmar que ha habido quienes han utilizado en forma inconfesada algunos referentes provenientes del discurso político, incluso en la elaboración de tesis doctorales de prestigiosas instituciones conservadoras. Ejemplo de ello es la incorporación, en el estudio de la subjetividad, de cierto tipo de literatura política que viene planteando la cuestión de la subjetividad desde el 
punto de vista de la psicología infantil, y que estaría presente no solamente en el individuo, sino también en el sujeto social que es su estado fundacional (en términos de Alberoni). Esto nos hace recordar aquella fuerza o masa (en la que pensaba Rosa Luxemburgo) que no se agotaba en ninguna de sus formas de expresión, pero que formaba parte de los procesos que fueron destacados en las distintas reflexiones teóricas: la interiorización y la subjetivización de lo social, y apunta con ello a las dos dimensiones de este continuo del cual partimos.

En este marco, resulta sorprendente abordar la gran discusión que, surgida a partir de la obra de Marcel Mauss, ha sido recuperada por varios autores, y entre los cuales destaca Dumont por sus aportaciones originales. Dumont planteó un conjunto de problemas relacionados con la dinámica que, en el transcurso de la historia, ha experimentado el proceso de constitución del individuo y de la sociedad. No es posible aquí detallar ese conjunto de consideraciones relevantes desarrolladas por él, sino tan sólo nombrarlas: me refiero, por ejemplo, al problema fundamental de la relación del individuo con el mundo, a partir del análisis de ciertos tipos de proceso de secularización; al respecto, podríamos afirmar que esa es una línea de pensamiento centrada en la clásica relación entre individuo y sociedad.

Hay igualmente otras discusiones, en la línea de recuperar el problema de eso que hemos denominado la subjetividad en el plano del individuo y en el plano de lo social; tal es el caso de autores como Lipovetski, que, a diferencia de los anteriores, no se concentra en el proceso de la individuación, sino en lo que dicho autor denominó la personalización. En la visión de Lipovetski hay una idea importante que en su momento tuvo tintes muy polémicos, y que está en el centro de la discusión de los estudios que han abordado el problema de las condiciones estructurales. Ahí el trasfondo apunta al hecho de que la dimensión de lo social en la actualidad tiene la característica de multiplicarse: lo social se multiplica y se diversifica y da paso a instituciones sociales flexibles en extremo; lo cual determina que el sujeto mismo, incluso como individuo, también se diversifique y se movilice. Planteamiento amplio y complejo que merece destacarse, en tanto incorpora en el debate sobre los sujetos sociales la significación, conferida en su análisis a las heterogeneidades presentes en la sociedad, las cuales influyen sobre el proceso de constitución de lo subjetivo. Idea importante, en tanto resignifica el dinamismo del contexto y reproblematiza el tema de las instituciones. Tal perspectiva podría también permitir enlazarse con vieja discusión determinista que provenía del pensamiento clásico en el orden político y en el cual se utilizaba una especie de lógica cartesiana.

En relación con el contexto -ya de por sí complejizado-, a pesar de ubicarlo en el plano de los condicionamientos estructurales, requiere indagar si tiene relevancia el presupuesto de ciertas homogeneidades sociales como la clase. Habría que preguntarse qué tiene que ver la clase con las consideraciones que apuntan a una mayor heterogeneidad del contexto. Debido a que la clase es una categoría y no un concepto teórico (con un contenido cerrado), el problema no está en la categoría, 
sino en la forma en que es razonada. El propósito es que dicha categoría sea capaz de rendir cuentas respecto de los condicionamientos estructurales, cuya complejidad está siendo puesta de relieve por una parte del discurso académico actual.

En este sentido se orienta la discusión abierta por Maffesoli, por ejemplo, cuando formula su idea sobre el neotribalismo, que apunta al surgimiento de nuevas formas de agrupamientos humanos que pueden tener cierta permanencia, pero que muy probablemente no ostenten la consistencia y la continuidad de las viejas organizaciones presentes en el llamado "momento" de la modernidad. Podría ser importante, desde luego, eso que se ha denominado "grupos afectivos" (sin ser espacios terapéuticos), cuya significación es de interés en el análisis de las dinámicas sociales más diversas, pues ello también daría cuenta de la complejidad inherente a las heterogeneidades de los contextos, como éstos están surgiendo en el presente.

Con la apertura de este conjunto de ideas, es necesaria su reapropiación en el marco que inicialmente diseñamos; es decir, en el horizonte de la vieja problemática del sujeto y de la subjetividad social, pues ello posibilita confrontar las concepciones dicotómicas con las que apuntan a la idea de continuo. Por ejemplo, ciertas afirmaciones de Maffesoli tienen diversas implicaciones, como aquella referida a las agrupaciones móviles fluidas que, sin duda, podrían permitir repensar muchos de los conceptos clásicos (de mayor dureza) provenientes de la teoría sociológica, por mencionar sólo una disciplina. En toda la teoría de los grupos probablemente estemos llegando a un punto en que la vieja conceptualización de los grupos primarios, secundarios o terciarios ya no tenga relevancia y que estemos, por tanto, ante el surgimiento de lo que hemos llamado las subjetividades sociales con o sin capacidad de construcción.

\section{Cinco líneas}

Si resumimos lo dicho hasta el momento, y que constituye un desafío para la construcción del análisis del cual venimos ocupándonos, plantearíamos cinco grandes líneas:

1. Primero apuntaría a una cuestión muy discutida y aún no resuelta: me refiero al problema y la necesidad de dar cuenta de los dinamismos constitutivos en los agrupamientos colectivos; de responder a la pregunta de cuáles son sus formas de expresión; de saber si la explicación de tal dinamismo se agotó en la imagen de las viejas estructuras conceptuales provenientes del continuo: clase-grupo primario y, por tanto, de si estamos enfrentados (como podrían estar sugiriéndolo algunos autores) al hecho de que habría que someter a revisión el conjunto de ese esquema conceptual.

2. La dificultad del análisis referido al dinamismo constitutivo de los colectivos o de los sujetos sociales no sólo se deriva de las complejidades múltiples y simultáneas que les son intrínsecas, sino sobre todo del hecho de que tales dinamismos conformadores de colectivos están influidos con mucha fuerza 
por las situaciones históricas y, por tanto, por la incorporación de los contextos históricos en el análisis de esos dinamismos; éste no es un problema puramente parametral o de ubicación, sino parte consustancial al problema tratado.

3. Hay dos pares de juegos conceptuales que podrían constituir el inicio (lo digo con mucha cautela) de una discusión más profunda de estos problemas: me refiero primero al viejo concepto de sociedad, que podría acompañarse con el añejo concepto de individuo y que, de manera simultánea, es posible que estén dando origen a determinados tipos de análisis en relación con lo social y con la subjetividad de lo social; estos conceptos posiblemente coinciden con los viejos esquemas de pensamiento que tuvieron presencia sobre todo en los grandes discursos políticos durante el siglo XX.

4. El segundo par de conceptos que han empezado a emerger, y que no estoy seguro lleguen a tener la relevancia de los anteriores, pero que habría que revisar en tanto han sido forjados con propósitos analíticos, son los conceptos de socialidad y personalización; tales conceptos plantean muchas interrogantes y sugieren líneas de razonamiento que, quizá, no sean en todo coincidentes respecto a los conceptos clásicos, en tanto sus pretensiones son en mayor medida de carácter analítico. No obstante, ambos discursos tienen el propósito de responder cabalmente a las siguientes preguntas: ¿Qué se entiende por subjetividad social? ¿Qué significa subjetivación de lo social? ¿Qué quiere decir constitución de las subjetividades sociales?

5. Finalmente, hay que plantear el problema de que la presencia de los sujetos sociales pone en cuestión algunos aspectos básicos en relación con su tratamiento: en primer lugar, pone en tela de juicio lo que podríamos definir como los parámetros de homogeneización, e impone, por tanto, los de heterogeneidad; al respecto, la relevancia de este problema consiste en que se cuestiona la pretensión de cientificidad del análisis de los sujetos sociales. Parece -manejemos esto como hipótesis- que el parámetro de la búsqueda de regularidades (que hasta ahora ha sido el parámetro de cientificidad del pensamiento sociohistórico) no tuviera una presencia importante. En su momento Gramsci trató de enfrentar este problema, al plantear la imposibilidad de establecer una ley que explicara la sociedad entera; ello en razón de que en la práctica reflexiva de Gramsci, estaba ya presente el problema de las heterogeneidades.

Es posible que a lo que estemos enfrentándonos sea simplemente a la "comprensión" de esa concepción envolvente, aquello que envuelva lo teórico, y que, de alguna manera, podría representar un concepto de ciencia como equivalente al de conciencia histórica. Desde luego, el concepto de "comprensión" lo entendemos 
en el sentido diltheyano antes que en la acepción weberiana; aunque también, lo asociamos con un concepto de ciencia inclusiva acuñado por Marx, que Manuel Sacristán ha comentado ampliamente en sus textos.

Como quiera que fuere, quizás el tema de los sujetos está poniéndonos frente al desafío que hemos bosquejado, evitando situarnos ante el dilema de encontrar o no la ley que rige el comportamiento de los sujetos. Lo cual nos hace pensar en una suerte de modalidad de "comprensión" del momento histórico para dar cuenta de su diversidad, expresando con ello algo distinto de la simple prueba de una hipótesis: posiblemente el secreto está en la capacidad del investigador de articular las diversidades entre sí, como éstas emergen en un momento dado del tiempo.

\section{Bibliografía}

Bahro, R. (1981). Por un comunismo democrático. Barcelona: Fontamara.

Bloch, E. (1977). El principio esperanza. Madrid: Aguilar.

Canetti, E. (2002). Masa y poder. Barcelona: Galaxia Gutenberg

Cerroni, U. et al. (1969). Teoría marxista del partido político. Cuadernos de pasado y presente 12. Ciudad de México: Siglo XXI Editores.

Lipovetski, G. (1988). La era del vacío: ensayos sobre el individualismo contemporáneo. Barcelona: Anagrama.

Lukács, G. (1967). Estética. Barcelona: Grijalbo.

Maffesoli, M. (1990). El tiempo de las tribus: el declive del individualismo en las sociedades de masas. Barcelona: Icaria.
Magri, L. et al. (1975). Movimiento obrero y acción politica. Ciudad de México: Era.

Mignolo, W. (2002). Colonialidad global, capitalismo y hegemonía epistémica. En C. Walsh et al. Indisciplinar las ciencias sociales. Quito: Abya-yalaUniversidad Andina Simón Bolívar.

Pannekoek, A. (1978). Derrumbe del capitalismo o sujeto revolucionario. Ciudad de México: Siglo XXI Editores.

Reich, W. (1973). La psicología de masas del fascismo. Ciudad de México: Martínez Roca.

Rossanda, R. (1975). De Marx a Marx. Barcelona: Anagrama.

Wallerstein, I. (1996). Después del liberalismo. Ciudad de México: Siglo XXI Editores.

Zemelman, H. (1992). De la historia a la politica. Ciudad de México: Siglo XXI Editores. 